\title{
Health care reform: the differential diagnosis
}

E conomic and political experts appear to agree that the US health care system is a horrible mess, and there is no consensus as to how to fix it. The differential diagnosis is that our health care system: (a) is dying because of hopelessly opposed political parties and greedy insurance companies or (b) has a treatable illness. I believe that the illness is treatable, if we adhere to several guiding principles. These include (a) universal access to compassionate health care, (b) improved health care through scientific advances, and (c) reduced administrative and legal costs. Establishing these three goals, and introducing a series of changes designed to achieve them, can result in meaningful health care reform.

Goal number one: universal, compassionate coverage. The first goal should be the easiest to achieve. Initially, through a combination of private and public options, everybody should have some form of health insurance. I believe that the ultimate goal should be a singlepayer system, but that need not be the first step toward universal coverage. Achieving universal health insurance today would require the government to provide coverage for that portion of the $15 \%$ in the United States who are currently uninsured and cannot afford health insurance and to make health insurance a requirement for those who can afford it. The reality is that everybody now has access to health care, but the uninsured primarily use the most expensive forms of health care (e.g., the emergency room), boosting the costs. Cost savings from regulating physician income and from administrative and tort reform could be used to pay for universal coverage. A bigger challenge is training the pri- mary care providers who will be required in a new, more efficient health care system. This will require increased efforts by US medical schools to recruit and train primary care providers. To promote preventive care, all insurers should be required to refund a portion of insurance costs to

\section{The physician's voice}

The reality is that everybody now has

access to health care, but the uninsured primarily use the most expensive forms of health care (e.g., the emergency room), boosting the costs.

patients who undergo their annual physical examinations. The discussion about health care has to refocus on how to provide access for all to a physician who is well trained and cares about patients and who works in a system that is humane and no longer treats patients and physicians like commodities.

Goal number two: scientific advances in medical knowledge. America has the best biomedical research infrastructure in the world. However, this great national treasure is fragile and must be continually supported in order to develop new, more effective and affordable diagnostics and therapies. Sustained increases in support for biomedical research are an essential component of health care reform.

Goal number three: reducing costs. Any discussion of meaningful health care reform has to include improvements in diagnosis and effectiveness of health care delivery achieved using electronic medical records. The costs of implementing electronic patient records should be shared among the medical schools, as a single format would meet the needs of all. Similarly, as a first step toward a single-payer plan, all insurance providers should be required to use the same form, which would largely be filled out automatically using the electronic record. This would drastically reduce administrative costs associated with insurance and billing. Additional cost savings could be achieved by meaningful tort reform that protects the legal rights of patients but reduces frivolous lawsuits. This could be facilitated by an enhanced system of case review by experts, prior to initiating costly legal proceedings. Further cost regulation should include compensating all physicians with salaries, which would remove incentives for unnecessary procedures.

The diagnosis is clear: our health care system is in critical condition. However, its strengths include the world's best systems for training health care providers and biomedical researchers. The quality and effectiveness of the administrative components of our health care system must be improved so that they enhance the health of the patient, rather than destroy it.

\section{Andrew R. Marks}

Columbia University, New York, New York, USA.E-mail: arm42@columbia.edu.

J. Clin. Invest. 119:2853 (2009). doi:10.1172/ JCI41022.

\section{An academic, apolitical approach to health care reform}

$T_{\text {h }}$ addressing one of the most important issues facing the country, its health care system. An underlying premise of the need for reform is a belief that all citizens of this great country should have access to excellent health care. To accomplish this, universal health insurance must be a component of any plan. Many controversial issues have arisen in the debate, most relating to how to pay for those presently uninsured. Opponents are concerned that this will require increased taxation and/ or decreased support for those presently insured. The only acceptable solution will 